\title{
Locally finite varieties of groups arising from Cross varieties
}

\section{Sheila Oates Macdonald}

Let $\underline{\underline{\mathrm{V}}}$ be a Cross variety and let $n$ be the least integer such that $\underline{\underline{V}}^{(n)}$ is locally finite; then $n \leq 2 d+3$ where $d$ is an upper bound for the number of generators of certain critical groups in $\underline{\underline{\mathrm{V}}}$.

\section{Introduction}

If $\underline{\underline{V}}$ is a Cross variety then, by the Oates-Powell Theorem, $\underline{\underline{v}}=\underline{\underline{V}}^{(n)}$ for some $n$ and hence $\underline{\underline{V}}^{(n)}$ is locally finite, but, of course,

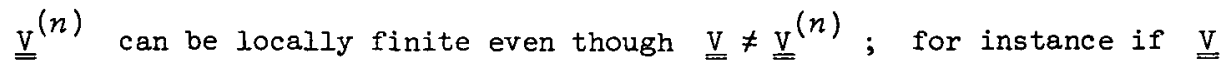
is the variety generated by the dihedral group of order $2^{r+1}$ then $\underline{\underline{v}}^{(2)}$ is locally finite, although $\underline{\underline{v}} \neq \underline{\underline{v}}^{r-1} \quad$ [4]. Can anything be said in general about the local finiteness of $\underline{\underline{V}}^{(n)}$ ? Certainly $\underline{\underline{V}}^{(1)}$ is not always locally finite [7], but I conjecture that $\underline{\underline{v}}^{(2)}$ is. Certain evidence to support this exists: R.M. Bryant [1] has shown that the two variable laws of $\operatorname{PSL}(2, q)$ imply local finiteness, thus extending the results of [2].

Both proofs of the Oates-Powell Theorem [8], [3] give values of $n$ for which $\underline{\underline{V}}^{(n)}$ is locally finite, though these tend to be somewhat large. In this paper I extend the results of [8], \$3, to prove:-

THEOREM A. Let $\underline{\mathrm{V}}$ be a Cross variety with a chain of subvarieties 


$$
\underline{\underline{\mathrm{E}}}=\underline{\underline{\mathrm{V}}}_{0} \subset \underline{\underline{\mathrm{V}}}_{1} \subset \ldots \subset \underline{\underline{\mathrm{V}}_{r}}=\underline{\underline{\mathrm{v}}},
$$

each maximal in the succeeding one, and let $\underline{\mathrm{V}}_{i}=\operatorname{var}\left(\mathrm{V}_{i-1}, D_{i}\right)$ where $D_{i}$ is critical and can be generated by $d$ (or fewer) elements; then $\underline{\mathrm{v}}^{(2 d+3)}$ is zocally finite.

\section{Notation}

Notation and terminology follow that of Hanna Neumann [6].

\section{Outline of proof}

It is clearly sufficient to prove the following theorem:

THEOREM B. Let $\underline{\underline{\mathrm{V}}}$ be a Cross variety and $\underline{\underline{\mathrm{U}}}$ a maximal subvariety of $\underline{\mathrm{V}}$ such that $\underline{\underline{\mathrm{V}}}=\operatorname{var}(\underline{\underline{\mathrm{U}}}, D)$ where $D$ is a critical d-generator group. If $\underline{\underline{\mathrm{U}}}^{(2 d+3)}$ is zocally finite so is $\underline{\underline{\mathrm{V}}}^{(2 d+3)}$.

Theorem A follows by induction on $r$ from Theorem $B$, since it is trivially true for $\underline{\underline{E}}$.

The proof of Theorem $B$ divides into two parts according as $\sigma D$ is abelian or non-akelian.

\section{4. $\sigma D$ abelian}

DEFINITION 4.1 . Let $\left\{W_{1}=1, \ldots, W_{k}=1\right\}$ be a basis for the $(2 d+3)$-variable laws of $\underline{\underline{U}}$ (by a result of B.H. Neumann [5] such a finite basis exists) and let $W(G)$ be the corresponding word subgroup, so that

$$
G / W(G) \in \underline{\underline{U}}^{(2 d+3)}
$$

and $G / N \notin \underline{\underline{U}}^{(2 d+3)}$ if $N<W(G)$. Similarly let $\left\{w_{1}=1, \ldots, w_{l}=1\right\}$ be $a$ basis for the $d$-variable laws of $\underline{\underline{U}}$, and $w(G)$ the corresponding word subgroup. Note that, since $\underline{\underline{\mathrm{U}}}^{(d)} \supseteq \underline{\underline{\mathrm{U}}}^{(2 d+3)}, w(G) \leq w(G)$.

LEMMA 4.2. If $G \in \underline{\underline{\mathrm{V}}}, w(G)=W(G)$.

Proof. Suppose there is $G \in \underline{\underline{V}}$ such that $w(G)<W(G)$, then, 
(*)

$$
\left\{\begin{array}{l}
G / w(G) \in \underline{\underline{\mathrm{V}} \cap \underline{\underline{\mathrm{U}}}^{(d)} \text { and }} \\
G / w(G) \mid \underline{\underline{\mathrm{V}}} \cap \underline{\underline{\mathrm{U}}}^{(2 d+3)} .
\end{array}\right.
$$

However

$$
\begin{aligned}
& \underline{\underline{\mathrm{V}}} \supseteq \underline{\underline{\mathrm{V}}} \cap \underline{\underline{\mathrm{U}}}^{(d)} \supseteq \underline{\underline{\mathrm{U}}},
\end{aligned}
$$

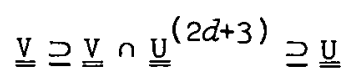

and $\underline{\underline{\mathrm{U}}}$ is maximal in $\underline{\underline{\mathrm{V}}}$.

Moreover $D \notin \underline{\underline{U}}^{(d)}$ (since it is a d-generator group not in $\underline{\underline{U}}$ ). It follows that

$$
\underline{\underline{v}} \cap \underline{\underline{U}}^{(d)}=\underline{\underline{\underline{U}}}=\underline{\underline{\mathrm{v}}} \cap \underline{\underline{\mathrm{U}}}^{(2 d+3)},
$$

contradicting $(*)$. Hence $w(G) \nmid W(G)$.

LEMMA 4.3. If $G \in \underline{\underline{\mathrm{V}}}$ then $W(G)$ is elementary abelian of exponent $p$, where $\sigma D$ is a p-group.

Proof. $D / \sigma D \in \underline{\underline{U}}$ (since $D$ is critical and $\underline{\underline{U}}$ is maximal in $\underline{\underline{V}}$ ) so $W(G) \leq \sigma D$. But $D \notin \underline{\underline{\mathrm{u}}}^{(2 d+3)}$ so $W(D) \neq 1$. It follows that $W(D)=\sigma D$ is elementary abelian, and $D$ satisfies the laws:

$$
\left[w_{i}, w_{j}\right]=1, w_{i}^{p}=1 \quad(i, j=1, \ldots, k)
$$

where the sets of variables in $W_{i}$ and $W_{j}$ are disjoint.

Since $\underline{U}$ also satisfies these laws, $\underline{\underline{\mathrm{v}}}$ must satisfy them and hence $W(G)$ is elementary abelian for every $G \in \underline{\underline{\mathrm{V}}}$.

COROLLARY 4.4. $\underline{\underline{\mathrm{v}}}$ satisfies the ((2d)-variable) lows $\left[w_{i}, w_{j}\right]=1, w_{i}^{p}=1 \quad(i, j=1, \ldots, 2)$.

Proof of Theorem B for abelian $\sigma D$. Let $G$ be a finitely generated group in $\underline{\underline{V}} \cdot G / W(G) \in \underline{\underline{U}}^{(2 d+3)}$ and so is finite. It follows that $W(G)$ is finitely generated and so is generated by a finite number of words of the form $w_{i}\left(g_{1}, \ldots, g_{2 d+3}\right)$.

Let $H=g p\left(g_{1}, \ldots, g_{2 d+3}\right)$; then $H \in \underline{\underline{\mathrm{V}}}$ and so $w(H)=\omega(H)$. Thus 


$$
w_{i}\left(g_{1}, \ldots, g_{2 d+3}\right) \in w(H) \leq w(G) .
$$

Hence $W(G)=w(G)$ and $w(G)$ is also finitely generated. But the laws $\left[w_{i}, w_{j}\right]=1$ and $w_{i}^{p}=1$ hold in $\underline{v}^{(2 d+3)}$ (being (2d)-variable laws of V). It follows that $w(G)$ is a finitely generated elementary abelian $p$-group and so is finite. Hence $G$ is finite as required.

\section{5. $\sigma(D)$ non-abelian}

Consideration of Section 3 [8] shows that, for the purposes of proving local finiteness $a$ (the number of variables in a basis for $\underline{\underline{U}}$ ) can be replaced by $2 d+3$ (the number of variables needed to ensure local finiteness) and $b$ (the size of a generating set for $D$ which includes one for $\sigma D$ ) can be replaced by at worst $d+1$, since it is sufficient to work with a generating set for $D$ which contains one element from $\sigma D$. Thus, by the results of $\$ 3.4$ of [8] we have that $\underline{\underline{V}}^{(n)}$ is locally finite, for

$$
\max (2 d+3,2(d+1)+1)=2 d+3
$$

\section{References}

[1] Roger M. Bryant, "On the laws of certain linear groups", J. London Math. Soc. (to appear).

[2] R.M. Bryant and M.B. Powell, "Two-variable laws for PSL(2, 5)", J. Austral. Math. Soc. 10 (1969), 499-502.

[3] L.G. Kovács and M.F. Newman, "Cross varieties of groups", Proc. Roy. Soc. Ser. A 292 (1966), 530-536.

[4] Sheila Oates Macdonald and Anne Penfold Street, "On laws in linear groups", (to appear).

[5] B.H. Neumann, "Identical relations in groups. I", Math. Ann. 114 (1937), 506-525.

[6] Hanna Neumann, Varieties of groups (Ergebnisse der Mathematik und ihrer Grenzgebiete, Band 37, Springer-Verlag, Berlin, Heidelberg, New York, 1967). 
[7] P.S. Novikov and S.I. Adjan, "Infinite periodic groups. I, II, III" (Russian), Izv. Akad. Nauk SSSR Ser. Mat. 32 (1968), 212-244, 251-524, 709-731.

[8] Sheila Oates and M.B. Powell, "Identical relations in finite groups", J. Algebra 1 (1964), 11-39.

University of Queensland,

St Lucia,

Queensland. 